\title{
RUPTURA DEL UTERO DURANTE EL TRABAJO DE PARTO
}

\author{
CASOS VISTOS EN EL HOSPITAL "MONFORT" DE VILLAVICENCIO
}

\section{Doctor José Alejandro Vega F.}

El presente trabajo no tiene tan sólo la finalidad de llenar un requisito. Siempre me ha llamado la atención ver la incidencia tan grande que tiene en esta región, la Rotura de Matriz durante el trabajo del parto y hemos podido ver que los casos que llegan al hospital con esta grave lesión ya no tiene otro tratamiento quie el extraer el feto muerto, anexos y sangre libre en cavidad abdominal, pues todos llegan con la rotura ya consumada. No pretendo en el presente trabajo sentar cátedra sobre estadísticas de traamientos, curaciones o muertes, pues tan sólo he revisado historias de cinco años y me parece que son pocos casos para una tarea de esta envergadura, que debe tener por demás muchisimos datos. Pretendo si dar a conocer de la Sociedad de Obstetricia y Ginecología este número de casos para que se tenga en cuenta la necesidad de una campaña a fondo de las autoridades sanitarias en el sentido de orientar los consultorios especialmente en los pueblos, hacia la consulta prenatal, educando a la futura madre y haciéndole ver el peligro a que está expuesta esperando su pequeño en el campio y sobre todo confiada a manos inexpertas. También tiene mucha importancia el hecho que algunas droguerías vendan sin mayores requisitos los ocitócicos, drogas que irán a aplicar las comadronas matando prácticamente a una parturienta, pues aunque no lo tenemos plenamente comprobado, sí nos da derecho a pensar que algo malo se ha hecho a una enferma que llega con la matriz rota, máxime si es multipara y no ha tenido ningún inconveniente en otros casos. Por todo lo anterior he escogido este tema para el presente trabajo que con toda honradez presento a la Sociedad de Obstetricia y Ginecología.

\section{DESGARROS O ROTURAS DEL UTERO}

Se designan bajo el nombre de roturas del útero, las soluciones de continuidad, que interesan toda la porción del órgano, situadas por encima de la inserción vaginal. Este grave 
accidente puede sobrevenir durante el embarazo o en el trabajo del parto; la muerte del feto es inevitable y de la madre si no se interviene rápidamente.

Las roturas uterinas son raras: hay autores que dan $\mathrm{I} \times \mathrm{I}$.000 otros $\mathrm{I} \times$ 3.000. Si se cuentan todos los desgarros incompletos o inadvertidos, sería mucho más la proporción; toda causa que adelgace las paredes uterinas, predispone a este accidente; la cicatriz de cesárea anterior, los abortos, la extirpación de miomas y sobre todo las infecciones.

Debemos distinguir los desgarros del segmento inferior y los desgarros del cuerpo del útero: como hemos visto es un accidente raro que puede presentarse en cualquier mes del embarazo, depende del medio ambiente donde se tome la estadística y del nivel cultural obstétrico de la región; en el caso del presente trabajo es más elevado, porque el Meta tan solo cuenta con el Hospital de Villavicencio, para atender casos quirúrgicos; aquí viene gente de todo el Departamento, del Vichada y parte del Casanare; en los pueblos tan sólo hay Puestos de Salul, donde no pueden atender ningún caso obstétrico complicado, en segundo lugar, en los pueblos y el campo la gente todavía le tiene recelo al médico, prefieren los servicios de una comadrona ignorante que armada de una jeringuilla aplica Pitocín y Ergotrate, sin pensar en las graves lesiones que ocasiona a la parturienta y ésta tan sólo recurre al Hospital cuando el caso es desesperado si es que ha tenido tiempo de hacerlo; ésta es la principal causa de la Rotura Uterina en esta región; en los centros de mayor cultura se ve menos precisamente por la previsión del accidente. Según Ritcher la Rotura Uterina en el parto se produce fundamentalmente por: a) Hiperdistensión de las paredes uterinas; b) por lesión de las mismas y otros autores añaden las traumáticas puras.

\section{ROTURAS UTERINAS POR HIPERDISTENSION}

La causa más importante es la estrechez pélvica, pero ha sido superada en nuestros tiempos por el uso incorrecto de los preparados de hipófisis posterior, el útero está dividido en tres partes de muy distinto fisiologismo: ia superior espesa y contráctil, es el cuerpo propiamente dicho, una zona intermedia delgada y flácida, es el segmento inferior y finalmente el cuello; la dilatación del cuello se hace por tracción excéntrica que sobre él realiza la zona contráctil, por intermedio del segmento inferior. El útero al contraerse tira del segmento inferior y se va engrosando mientras el segmento inferior se va adelgazando cada vez, más para lograr así la dilatación del cuello; el límite entre la zona contráctil y el segmento inferior es un rodete carnoso, el anillo de Bandl; en la inminencia de ruptura éste sube por encima del ombligo y se hace oblicuo. Cuando no puede progresar el feto, por estrechez pélvica, edema del cuello, presentación 
viciosa o cualquier otra distocia, el segmento inferior se va adelgazando al máximo, cede y se rompe. En la ruptura uterina por lesión de las paredes, la más común es la que se hace por la cicatriz de una cesárea anterior; también hay que tener en cứcnta los raspados uterinos, la multiparidad y especialmente las infecciones endouterinas.

\section{ROTURAS UTERINAS TRAUMATICAS}

La que da más contingencias es la versión y es debida a la distensión que sufre el segmento inferior al introducir profundamente la mano para hacer presa del pie, olvidando fijar con la otra mano el fondo del útero; en nuestro caso figura una paciente con ruptura uterina por arma de fuego.

\section{Anatomía patológica}

Las rupturas uterinas pueden ser completas o incompletas.

\section{Ruptura completa}

Lo característico pero no constante, es el paso de sangre, cuerpo del feto y placenta a la cavidad abdominal; el orificio de ruptura es anfractuoso, con bordes sembrados de hematomas; depende de esta brecha el tratamiento conservador o no; cuando se ha interesado la bóveda vaginal y la vejiga, el caso es más complicado.

\section{Rotura incompleta}

En éstas el peritoneo queda íntegro, formando un toldo a la masa hemo- rrágica y a las partes del huevo que salen del útero; estas roturas incompletas predisponen a las roturas completas en partos posteriores.

\section{Sintomas de la rotura del útero}

La paciente está agitada, ninguna posición le acomoda, el hipogastrio es tenso y doloroso, el anillo de Bandl ha ascendido hacia el ombligo; en el examen interno encontramos la pelvis vacía y el cuello grueso y edematoso; esta situación ya no debe verse si la paciente está controlada por el tocólogo. En la rotura consumada encontramos una paciente en mal estado general, con shock, vómito, sudor frío, taquicardia y taquipnea, se palpan partes fetales a través de la piel del abdomen, no hay ruidos fetales y por la vulva sale una discreta hemorragia. Todo este cuadro lo vemos donde la consulta prenatal es nula, para prevenirlo se deben orientar los programas sociales en el sentido de la protección de la maternidad, muy especialmente facilitando a la embarazada la asistencia y vigilancia en los Puestos de Salud u Hospitales, pues la rotura uterina es hoy accidente de parto domiciliario y mal dirigido; toda paciente que haya sido sometida a cesárea, miomectomía, o sufrido una rotura anterior debe estar con tiempo en un ambiente clínico.

\section{Tratamiento}

Como primera medida controlar el estado de shock, con transfusiones, sueros, oxígeno, tonicardiacos, anti- 
bióticos para ir controlando la infección. En nuestros casos la cantidad de sangre transfundida fue pequeña porque el hospital no contaba con Banco de Sangre, pero es un detalle que no debe olvidarse. La intervención quirúrgica, laparatomía se practica bajo anestesia general, se extrae el feto, placenta y sangre y coágulos; se limpia bien la cavidad abdominal y se reconoce la brecha. Es bưena intención la de conservar en lo posible el órgano uterino; cuando la vitalidad del útero sea muy precaria, por zonas grandes de necrosis, hacer la histerectomía subtotal o total según el caso; en los desgarros del segmento en que la vitalidad del útero no está muy afectada, se sutura la brecha en tres planos con catgut cromado número I. En fin, todo esto depende del buen juicio del cirujano al analizar la situación; de resto el tratamiento médico general que exija cada caso, no descuidando los antibióticos ni el estado anémico de la paciente.

Historia Clínica № 207. Nombre: P. O. Edad: 26 años. Estado civil: casada. Ocupación: Of. domésticos. Procedencia: Granada-Meta. Fecha de entrada: marzo 3I/58. Diagnóstico probable: rotura uterina. Antecedentes familiares: sin importancia. Antecedentes obstétricos: partos 4 abortos o. U. R.: julio 5 ; meses de gestación: 9. Ruidos cardíacos fetales: negativos. Empezó trabajo en la casa, atendida por comadrona el 30 de marzo, 9 a. m. T. A.: 8 x 6. Pulso: roo. Piel y mucosas: piel pálida y sudorosa, mucosas pálidas. Digestivo: meteorismo, vó- mito y náuseas. Respiratorio: normal. Examen obstétrico.

A la inspección abdomen globuloso, secresión sanguinolenta por la vulva. A la palpación se encuentran partes fetales en abdomen. Tumoración con una escotadura del lado derecho. Al tacto cuello blando $4 \mathrm{ctm}$. de dilatación, se toca una brecha por encima del cuello; se inicia el tratamiento con transfusión de $500 \mathrm{cc}$. de sangre total, I.500 cc. de Dextrosa al $5 \%$ en agua, Terramicina $500 \mathrm{mlg}$. Se pasa a la Sala de Cirugía y bajo anestesia general laparotomía media infraumbilical. Se encuentra el feto y placenta libres en cavidad abdominal, sangre libre y coágulos en gran cantidad. Una ruptura del segmento que interesó cuello y parte de cúpula vaginal, el feto mostraba equimosis en hombro y dorso, muerto masculino de 3.600 grms. de peso. Se suturó la brecha en dos planos, con catgut cromado; el músculo y con simple serosa; los demás planos con seda $\mathrm{N}^{\circ}$ I. El postoperatorio no tuvo complicaciones, se aplicó Omnamicina 8 x I № 6, calmantes. La enferma salió del hospital en buenas condiciones el 9 de abril de 1958 .

Historia Clínica № 626. Nombre: C. H. Estado civil: soltera. Edad: 40 años. Ocupación: Of. domésticos. Procedente: Acacías. Antecedentes Obstétricos: 6 partos. Diagnóstico: ruptura uterina. Fecha de entrada: Sep. 24/59. U. R.: diciembre; meses de gestación: 9. Ruidos cardíacos fetales: ne- 
gativos. Edemas: no. Vómitos: no T. A.: $6 \times 4$. Pulso 90 .

Al examen general, se encuentra abdomen meteorisado y defendido, náuseas. Se palpan partes fetales por sobre la piel del abdomen. Al tacto, cuello grueso, edematoso $3 \mathrm{ctm}$. de dilatación, secreción sanguinolenta por vagina. Se aplica transfusión de 500 cc. Graplasmoid. Bajo anestesia general laparatomía media infraumbilical, se encuentra feto y libre en cavidad abdominal, lo mismo que placenta, sangre y coágulos. Se encuentra un desgarro de segmento inferior con lesión uterina izquierda. Se repara la brecha. La enferma muere seis horas después en shock.

Historia Clínica № 63. Observación personal. Nombre: E. A. Edad: 28 años. Estado civil: casada. Procedente: San Martín. Ocupación: Of. domésticos. Fecha de entrada: febrero 29/60. Diagnóstico: ruptura de matriz. Antecedentes obstétricos: partos 5; abortos o. U. R.. no sabe. Ruidos fetales: negativos. T. A.: c x o. Pulso: filiforme. Inicia trabajo el 28 en la casa bajo el cuidado de una comadrona.

Examen obstétrico: A la inspección se notan dos tumoraciones en abdomen. A la palpación se tocan partes fetales y una tumoración a la derecha. Por vulva sale secresión sanguinolenta. Al tacto se reconoce una brecha que arranca de cúpula vaginal hacia arriba. Se aplica transfusión de 500 cc. de sangre, Dextrosa en Sal. Salina Ioo cc. y en agua 500 cc. Terramicina
500 mlg. Con T. A. $8 \times 6$ se pasa a la Sala y bajo anestesia general laparotomía media infraumbilical. Se encuentra feto y placenta libres en cavidad abdominal, sangre cuágulos y meconio. Se extrae el feto y se limpia cavidad. Se reconoce una brecha que interesa cúpula vaginal, cuello y segmento. La uterina izquierda desgarrada y con un trombo, la matriz con zonas necróticas grandes. Se hace histerectomía subtotal. La enferma fallece cinco horas después en shock. El feto muerto masculino de 4.200 grms. con equimosis en hombro y tronco.

Historia Clínica № 554 . Observación personal. Nombre: N. R. Edad: 24 años. Procedente: San Martín (Me ta). Ocupación: Of. domésticos. Fecha de entrada. agosto 26/60. Diagnóstico: Ruptura de matriz, por presentación de hombro. Obstétricos: partos 2; abortos o. U. R. noviembre. Meses de gestación: 9. Edemas: no. T. A.: го x 8. Pulso: iпo. Ruidos cardíacos fetales: negativos. Piel y mucosas: piel pálida, sudorosa, mucosas pálidas. Digestivo: meteorismo, náuseas, tuvo vómitos por la mañana. Empezó trabajo en la casa el 24 de agosto por la tarde, bajo el cuidado de una comadrona.

Examen obstétrico: A la inspección abdomen globuloso, se palpa una tumoración con una escotadura del lado derecho, en la vagina hay procidencia de mano. Se aplica transfusión de de sangre total $500 \mathrm{cc}$. Terramicina 500 mgls. Se pasa a la Sala y bajo anestesia general laparotomía media infraumbilical. 
Se encuentra feto y placenta libres en cavidad abdominal, sangre y coágulos en cantidad apreciable. Extraído el feto, la placenta y limpia la cavidad abdominal, se reconoce la brecha que interesó cúpula vaginal, cuello y segmento; la matriz tenía zonas necróticas por lesión de los vasos uterinos. En estas condiciones se hace histerectomía subtotal. El postoperatorio fue accidentado, la enferma se meteorizó, se aplicaron sueros, Terramicina de 500 mgls. 5 fcos., Prostigmina, sonda gástrica y calmantes. Sale del hospital el 6 de Sept./6o, en buenas condiciones.

Historia Clínica № 687. Observación personal. Nombre: C. R. G. Edad: 27 años. Estado civil: casada Ocupación: Of. domésticos. Procedencia: Cubarral (Meta). Fecha de entrada: Octubre 2/60. Diagnóstico: ruptura de matriz. Antecedentes obstétricos: partos 2; abortos I. U. R.: diciembre. Meses de gestación: 9 . Ruidos cardíacos fetales: negativos

Examen obstétrico: abdomen globuloso, se tocan partes fetales y una tumoración con una escotadura. Secreción sanguinolenta por vagina. Cuello grueso, edematoso, $4 \mathrm{cmt}$. de dilatación. No se encuentra presentación. Empezó trabajo en la casa el $\mathbf{I}^{0}{ }^{\circ}$ de octubre, después el 2, sintió un dolor muy grande en abdomen y le pasaron los dolores, pero se sintió muy débil. Diámetros: Conj. ext. 19; B. C. 30; B. T. 32 ; B. E. 24. Digestivo: Meteorismo, náuseas, vómitos. Circulatorio: T. A.: 8 x 6. Pulso: i2o. Respiratorio: normal. Neuropsíquico: embotamien- to. Piel y musosas: piel pálida, sudorosa, mucosas pálidas.

Se aplica transfusión de 500 cc. de sangre total. Dextrosa en Sol. Salina I.600 cc. Terramicina x $500 \mathrm{mgls}$.

Bajo anestesia general, laparotomía media infraumbilical: se encuentra feto muerto y libre en cavidad abdominal, sangre y coágulos en cantidad. Matriz retraída y una brecha en segmento inferior cara anterior y borde izquierdo. Feto 3.80o. Se repara la brecha en tres planos. El postoperatorio fue normal. Se aplicó Penicilina diariamente. Terramicina y calmantes; la enferma abandona el hospital el I4 de octubre en buenas condiciones.

Historia Clínica ํo 9ıо. Nombre: H. de M. Estado civil: casada. Ocupación: Of. domésticos. Procedencia: Hato Corozal. Fecha de entrada: diciembre 3I/60. Edad: I7 años. Antecedentes obstétricos: partos I; abortos o. Diagnóstico: ruptura de matriz, por trabajo de parto mal dirigido en el campo. U. R.: 25 de octubre; meses de gestación: 9. Ruidos cardíacos fetales: negativos. Edemas: no. T. A. 8 x 6. Pulso: iıo.

Al examen clínico, abdomen meteorizado, muy doloroso, se palpan partes fetales y una tumoración a la derecha. Al tacto se encuentra cuello grueso, edematizado con tres cmts. de dilatación y secreción sanguinolenta por vagina. Se aplica transfusión 500 cc. Graplasmoid un fco. Bajo anestesia general laparotomía media umbilical. Se encuentra feto muerto, libre 
en cavidad abdominal, placenta libre en cavidad, sangre y coágulos. Matriz retraída y una brecha en cara anterior de segmento inferior. Se repara la brecha en tres planos. La enferma muere al segundo día por embolia pulmonar.

Historia Clínica No 646 . Observación personal. Nombre: M. de B. Edad: 40 años. Estado civil: casada. Ocupación: Of. domésticos. Procedencia: Cubarral. Fecha de entrada: julio I6/6r. Antecedentes obstétricos: partos 8 ; abortos o. Diagnóstico: ruptura uterina.

Tuvo el parto el i5 por la noche en la casa, llega al hospital el I6 por la noche en estado anémico y meteorizada, con un dolor muy fuerte en abdomen, náuseas y vómito; a la palpación se toca una matriz retraída pero muy dolorosa. Al tacto se encuentra cuello edematoso y con una brecha en labio anterior que se continúa hacia arriba. Dice la paciente que el feto nació muerto, después de más de un día de dolores. Se aplica transfusión de $500 \mathrm{cc}$. de sangre, Dextrosa en Sol. Salina y en agua 500 c/u. Terramicina Bajo anestesia general, se hace laparotomía media infraumbilical, se encuentra sangre libre y coágulos en cavidad abdominal; un desgarro de cuello y segmento inferior, longitudinal por el borde izquierdo, placas de peritonitis y matriz retraída. Se repara la brecha en tres planos. El postoperatorio fue accidentado; se meteorizó la enferma. Se aplicó Dextrosa en agua y Sol Sa- lina, Graplasmoid, Neostignina y antibióticos tipo Penicilina y Tetraciclina

La enferma abandona el hospital el 25 de julio en buenas condiciones.

Historia Clínica Nọ 8o6. Observación personal. Nombre: A. A. Estado civil: viuda. Edad: 25 años. Ocupación: Of. domésticos. Procedencia: Castilla La Nueva. Fecha de entrada: agosto 3/6r Diagnóstico: ruptura uterina. Antececienes obstétricos: partos 3; abortos o. U. R.: noviembre. Meses de gestación: 9 T. A.: i I x 7. Pulso: I40. Inicia trabajo el 2 de agosto en la casa a las 8 a. m.

En examen se encuentra abdomen globuloso, muy doloroso y una tumoración por debajo de partes fetales. Al tacto se encuentra el cuello desgarrado y una brecha que se continúa hacia arriba; secreción sanguinolenta y escasa por vagina. Ruidos fetales: negativos. Digestivo: meteorismo, vómito. Circulatorio: pulso filiforme aunque la tensión está en II x 7. La enferma se ve muy grave. Respiratorio. Cheyne Stokes. Neuropsíquico: embotamiento. Piel y mucosas: piel pálida y sudorosa, mucosas pálidas. Se aplica transfusión por $500 \mathrm{cc}$. Y bajo anestesia general, laparotomía media infraumbilical. Se encuentra feto muerto y libre en cavidad abdominal, placenta libre en cavidad, sangre y coágulos. Matriz retraída. Se reconoce un desgarro que interesa cuello, segmento inferior y parte del cuerpo. La paciente muere en la mesa de operaciones por shock. 
Historia Clínica № 818. Observación personal. Nombre: R. R. Estado civil: casada. Edad: 30 años. Ocupación: Of. domésticos. Procedencia: Acacías. Fecha de entrada: Sept. 6/6r. Antecedentes obstétricos: G: 2. el primer embarazo terminó con cesárea Abortos: o. U. R.: I $^{0}$ de diciembre Meses de gestación: 9. Diagnóstico: ruptura de matriz. Ruidos cardíacos fetales: negativos. T. A. Io x 9, pulso ıoo. Inicia trabajo el 5 de septiembre en la casa.

A la inspección se encuentra abdomen globuloso, partes fetales y una tumoración al lado derecho. Al tacto se encuentra cuello grueso, edematoso y secreción sanguinolenta por vagina. Pelvis vacía. Diámetros: C. E. I8; B. T. 3I; B. C. 27 ; B. E. 23. Se aplica transfusión por $500 \mathrm{cc}$. bajo anestesia general, laparotomía media infraumbilical. Se encuentra feto libre en cavidad abdominal, placenta desprendida y libre en cavidad, sangre y coágulos. La matriz desgarrada por la cara anterior del segmento precisamente por la cicatriz de la cesárea anterior. Se limpian los bordes y se suturan en tres planos. Feto muerto 3.500 grms. En el postoperatorio. Diallerpen, Terramicina. Calmantes. La enferma abandona el hospital en buenas condiciones el I4 de septiembre de ig6r.

Historia Clínica № 865. Observación personal. Nombre: B. de D. Estado civil: casada. Edad: is años Ocupación: Of. domésticos. Procedencia: Restrepo. Fecha de entrada:
Sept. 2I/62. Diagnóstico: ruptura de matriz en trabajo de parto en el campo. Antecedentes obstétricos: partos I. Abortos o. U. R.: r8 de diciembre. Meses de gestación: 9. T. A.: 8 x 5 . Pulso: i2o. Diámetros: C. E. I9; B. T. 32 ; B. C. 29 ; B. E. 24 . Ruidos cardíacos fetales: negativos. Digestivo: náuseas, vómito. Respiratorio: normal Neuropsíquico: Embotamiento. Piel y mucosas: piel pálida, sudorosa, mucosas pálidas. Inicia trabajo el 20 en la casa a las 6 a. m., bajo los cuidados de un comadrona.

Se aplica transfusión x 500 cc. Dextrosa en Sol. Salina. Bajo anestesia general, laparotomía media infraumbilical. Se encuentra feto muerto y libre en cavidad, placenta desprendida y libre en cavidad, sangre y coágulos. Se reconoce un desgarro de segmento y una brecha hacia cuerpo por el borde izquierdo. Se sutura la matriz en tres planos. Feto muerto 3.500 grms. bosa parietal. El postoperatorio no fue normal: la enferma hizo tromboflebitis, se trató con Liquemine, antibióticos, sueros. Deja el hospital el Io de octubre en buenas condiciones.

Historia Clínica № ro83. Nombre: S. B. Estado civil: casada. Edad: 2 I años. Ocupación: Of. domésticos. Procedencia: Villavicencio. Fecha de entrada: diciembre 3/62. Diagnóstico: ruptura de matriz en trabajo de parto. Antecedentes obstétricos: G. 4 P. 3. A. o. U. R.: febrero. Meses de gestación: 9. T. A.: 8 x 6 . Diámetros: C. E. 19 ; B. T. 3 I ; B. E. 24 ; B. C. 29. Ruidos cardíacos fetales: negativos. 
Cuello blando, dilatación completa, presentación insinuada, gran edema de vulva, secreción sanguinolenta por vagina, presentación cefálica. Empezó trabajo el 2 en la casa, la acompañó una enfermera. El 3 sintió un dolor muy fuerte en el bajo vientre y los dolores del parto le pasaron, pero estaba muy adolorida del abdomen; a la palpación no permite que la toquen, está agitada y sudorosa. Con cuidado se palpan partes fetales. Se aplica transfusión x 500. Dextrosa en Sol. Salina. Bajo anestesia general laparotomía media infraumbilical. Se encuentra feto libre en cavidad abdominal, lo mismo que placenta, sangre y coágulos. Se ve un estallido del segmento inferior y la uterina derecha desgarrada, se liga y se sutura la matriz en tres planos. Feto muerto 3.coo grms. La enferma muere al tercer día en shock. Se aplicaron antibióticos, sueros. etc.

Historia Clínica № I 137. Nombre: V. de B. Estado civil: casada. Procedencia: Acacías. Edad: 22 años Ocupación: Of. domésticos. Fecha de entrada: diciembre 12/62. Diagnóstico: heridas abdominales con arma de fuego, carga múltiple en gestante de seis meses. Antecedentes obstétricos: G. 8. P. 7. A. o. T. A.: 8 x 6. Pulso: I4?. Ruidos cardíacos fetales: negativos.

Presenta herida en flanco derecho y a nivel de la espina ilíaca derecha, por orificio grande. En flanco izquierdo dos orificios de salida. Digestivo: meteorismo, náuseas. vómito, de- fensa abdominal. Circulatorio: shock. Respiratorio: Cheyne Stokes.

Se aplica transfusión de 500 cc. Dextrosa en Sol. Salina r.000 cc. Velaciclina. Bajo anestesia general, laparotomía media infraumbilical, se encuentra sangre libre en cavidad abdominal. Dos asas delgadas desgarradas y el fondo de la matriz desgarrado, haciendo protución un pie destrozado del feto. Se extrae feto muerto masculino, I.500 grms. La matriz se sutura, se hace resección intestinal. En el postoperatorio se aplican antibióticos, Dextrosa en agua y Sol. Salina, calmantes. La paciente deja el hospital en buenas condiciones el 21 de diciembre de 1962.

Historia Clínica № II I2I. Observación personal. Nombre: A. G. Estado civil: soltera. Edad: 35 años. Ocupación: Of. domésticos. Procedencia: San Martín. Fecha de entrada: diciembre i8/62. Diagnóstico: ruptura de matriz por presentación de hombro. trabajo de parto en el campo. Antecedentes obstétricos: G. 5, P. 4. A. o. Diámetros: C. ex.: 20; B. T. 32 ; B. C. 28 ; B. E. 24 . T. A.: $6 \mathrm{x}$ 4. Estado psíquico: inquietud, embotamiento. Empieza trabajo en la casa el i6, bajo los cuidados de una partera, los dolores muy seguidos y el niño no nacía, la mujer le ayudó y no pudo; luego al tercer día no sentía al niño y se calmaron los dolores del parto, se sintió muy mala.

Examen obstétrico: Se encuentra mano en vagina, abdomen distendi- 
do, se aprecian partes fetales y una tumoración a la derecha. Se aplica transfusión de 500 cc. Dextrosa en agua y en Sol. Salina r.ooo cc. Bajo anestesia general, laparotomía media infraumbilical, se encuentra feto de 3.500 grms. muerto y libre en cavidad abdominal, placenta desprendida, sangre y coágulos; placas de peritonitis. Un desgarro de cúpula vaginal, cuello y segmento inferior; se sutura la brecha, en tres planos. Se aplica diariamente Velacicline $\mathrm{x} 700$ mgls. Dextrosa en Sol Salina y en agua, calmantes. La paciente muere al cuarto día por peritonitis.

\section{CONCLUSIONES}

I). Se estudiaron las Historias Clínicas de las pacientes que ingresaron al Servicio de Maternidad por embarazo a término, durante los años de 1958, I959, 1960, 1961 y 1962; fueron en total 4.204, de éstos ingresaron I3 casos de RUPTURA UTERINA.

2). Doce de estos casos presentaron la ruptura uterina durante el trabajo de parto, atendido en la casa a manos de comadrona.

3.) Se presentó un caso de ruptura uterina traumática por arma de fuego.

4). La proporción de casos por ruptura uterina durante el trabajo de parto fue de 2.85 por mil.
5). La proporción de casos de ruptura uterina traumática fue de 0,26 por mil.

6). En todos los casos se encontró feto muerto.

7). En todos los casos de ruptura uterina durante el trabajo de parto se encontró placenta libre en cavidad abdominal.

8). En todos los casos de ruptura uterina durante el trabajo de parto se encontró sangre libre y coágulos en cavidad abdominal.

9). En todos los casos de ruptura uterina durante el trabajo de parto se palparon partes fetales por debajo de la piel del abdomen.

I0). En todos los casos de ruptura uterina el estado de shock fue manifiesto.

II). En todos los casos de ruptura uterina se hizo laparotomía media infraumbilical bajo anestesia general.

I2). En todos los casos se trató de multíparas.

13). Un solo caso tenía antecedente de cesárea anterior. 0,26 por mil.

I4). Un solo caso da el antecedente de aborto. 0,26 por mil.

I5). La proporción de presentación de hombro y cefálica fue igual 
16). En seis casos se encontró lesión del cuello. 46,I por ciento.

I7). En dos casos hubo lesión del cuerpo. $15,3^{9}$ por ciento.
I8). De los trece casos vistos murieron seis pacientes. 46, Io por ciento.

\section{BIBLIOGRAFIA}

DEXEUS: Tratado de obstetricia.

NUBIOLA Y ZARATE: Tratado de obstetricia.

DELeE GRENhiLL: Principios práctica de obstetricia.

V. CONILL: Tratado de ginecología. 